\title{
Degeneracy, as opposed to specificity, in immunotherapy
}

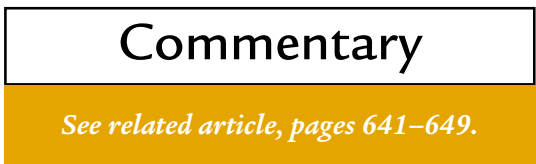

\author{
David A. Hafler
}

Center for Neurologic Disease, Brigham and Women's Hospital and Harvard Medical School, Harvard Institutes of Medicine, Room 786, 77 Avenue Louis Pasteur, Boston, Massachusetts 02115, USA. Phone: (617) 525-5330; Fax: (617) 535-5333; E-mail: dhafler@rics.bwh.harvard.edu.

J. Clin. Invest. 109:581-584 (2002). DOI:10.1172/JCI200215198.

While it could be argued that Jenner, with his discovery of vaccination, ushered in the field of immunotherapy, manipulation of the human immune system to treat inflammatory diseases is only now coming of age. The mechanism behind immune specificity, the basic premise of vaccination, was largely solved with appreciation of how the MHC presents antigen, and with crystallization of the MHC/T cell receptor complex. However, as has only recently been appreciated, while a given $\mathrm{T}$ cell receptor may ordinarily exhibit exquisite specificity, binding to some antigenic sequences can effect a conformational change to the receptor, allowing it to recognize MHC class II/peptide complexes in a highly degenerate fashion.

This capacity may represent a fundamental mechanism of autoimmune disease, since it allows microbial antigens to trigger the $T$ cell receptors of autoreactive $T$ cells. Indeed, as discussed below, the activation of these $T$ cells probably occurs both indirectly, by means of the innate immune system, and directly, through $T$ cell receptor stimulation (1).
Fortunately, the T cell receptor's degeneracy may be key to therapies, with advantages not seen with antigen-specific approaches. A recent series of publications offers new insights into how this aspect of the cellular immune response might be used to treat human autoimmune disease. One well-established but still only incompletely understood reagent that may work through such a mechanism is glatiramer acetate (GA) (Copaxone, registered trademark of Teva Pharmaceutical Industries Ltd.), discussed by Karandikar et al. in this issue of the JCI (2).

\section{$T$ cell receptor degeneracy}

Historically, the ability to generate and study cloned $\mathrm{T}$ cells reactive to self antigens reinforced the image of $T$ cell receptor interactions as highly specific for individual antigens. For example, $\mathrm{T}$ cell clones isolated from the blood of patients with multiple sclerosis (MS) exhibit exquisite specificity for the immunodominant p85-99 epitope of myelin basic protein (MBP) (3). However, this specificity is not absolute. Changing the $\mathrm{T}$ cell receptor contact residue lysine at position 93 to an arginine, or even just removing a hydroxyl group by changing a phenylalanine to a tyrosine at position 91 , can totally ablate $\mathrm{T}$ cell reactivity. Surprisingly, however, the lysine-to-arginine substitution also results in a more degenerate pattern of recognition by the $\mathrm{T}$ cell receptor, in that a tyrosine or other amino acid residues can now be tolerated at positions 91 or even 90 (4). Thus, while a T cell receptor appears to be highly specific in one situation, altering the peptide ligand can change the TCR conformation to yield a higher degree of $\mathrm{T}$ cell cross-reactivity.

Work using combinatorial chemistry to analyze another series of MBP p85-99-reactive $T$ cell clones leads to a similar conclusion. Hemmer et al. (5) identified a number of viral epitopes that can trigger autoreactive $\mathrm{T}$ cell clones in a manner that would not be predicted by simple algorithms. Indeed, one MBP-reactive $\mathrm{T}$ cell clone studied by these authors recognized an epitope of a different self protein entirely, the myelin oligodendrocyte glycoprotein.

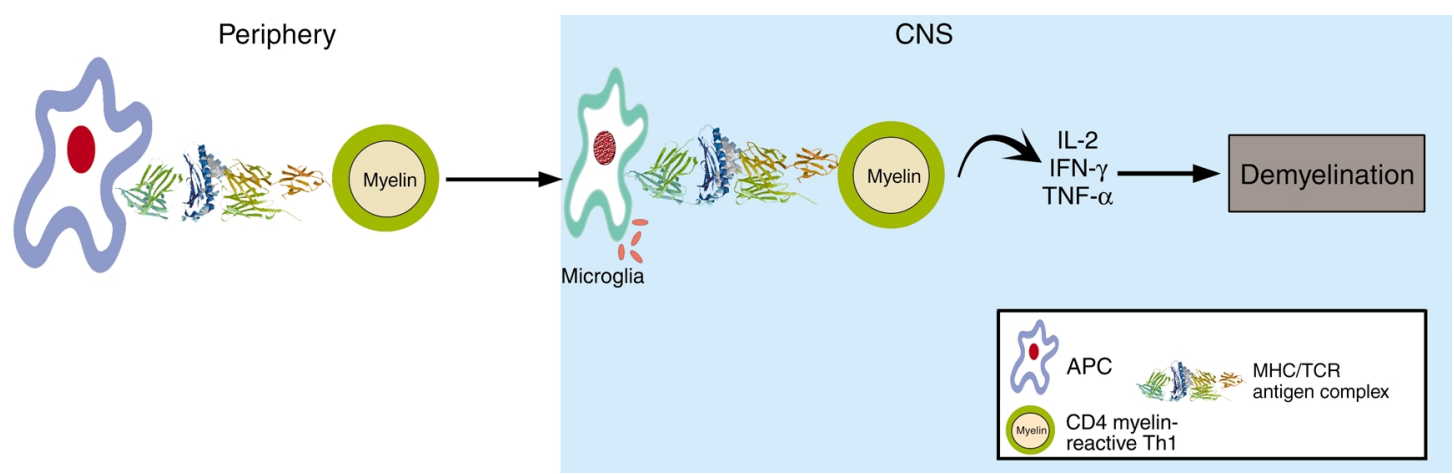

\section{Figure 1}

Autoimmune mechanism of MS. Naive, myelin reactive T cells are activated by microbes that: 1 ) induce the innate immune system to provide costimulatory signals necessary for clonal expansion of naive CD4 $4^{+} T$ cells and, 2$)$ contain epitopes that are cross-reactive with self antigens. Activated $C D 4^{+}$ $T$ cells cross the blood-brain barrier and recognize self antigen presented by microglia, local antigen-presenting cells (APC) that sample self antigens. The reactivation of autoreactive $\mathrm{CD}^{+}$cells leads to a cascade of events including recruitment of macrophages, antibody secretion, and eventual destruction of myelin and axons. 


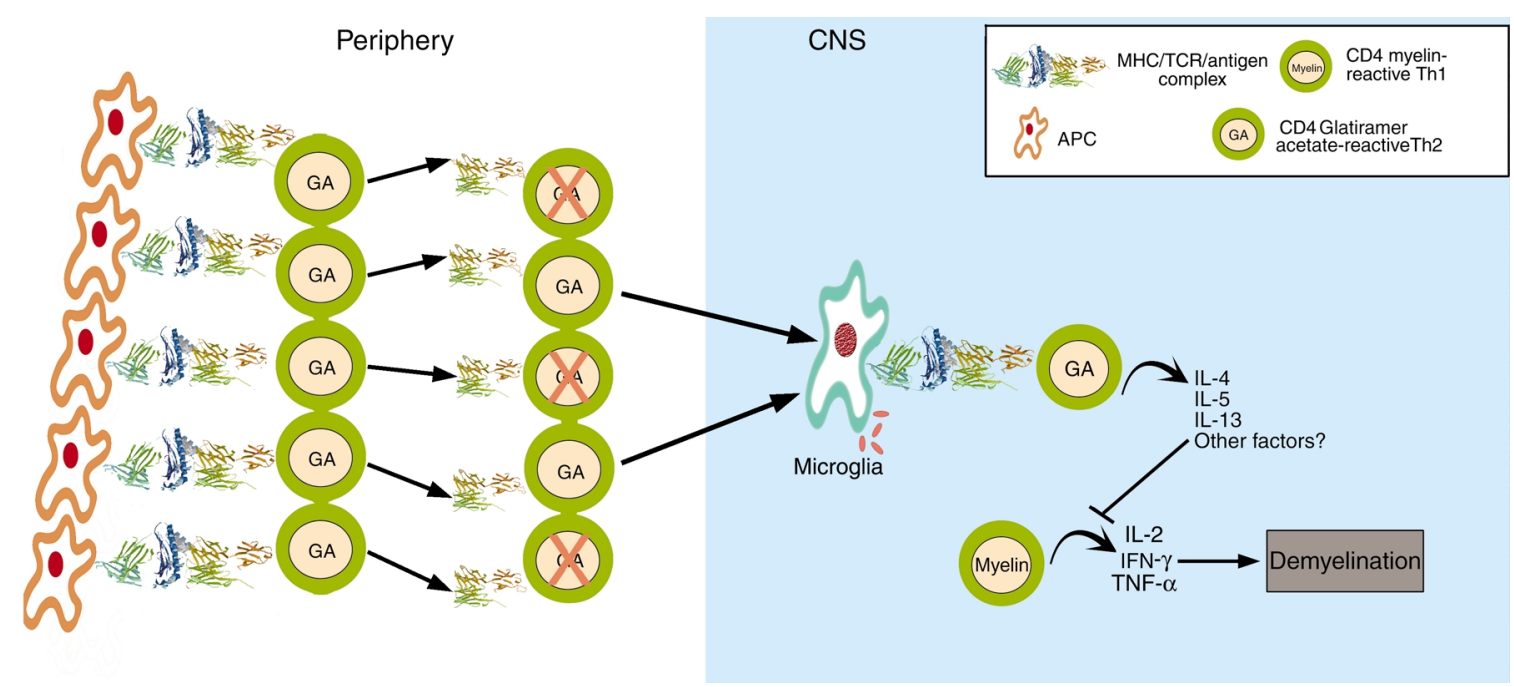

Figure 2

The $\mathrm{CD} 4^{+} \mathrm{T}$ cell model of action by glatiramer acetate (GA). GA induces strong MHC class II restricted proliferative responses by T cells. Daily injections of GA induces a moderate loss of responsiveness to the antigen accompanied by a shift to a more Th2 type of CD4 ${ }^{+} \mathrm{T}$ cell. The surviving GAreactive $T$ cells show a greater degree of degeneracy, as measured by cross-reactive responses to combinatorial peptide libraries. Highly cross-reactive Th2 cells with degenerate T cell receptors migrate to the site CNS, recognize self antigens as weak agonists or "altered peptide ligands". In response, they begin to secrete Th2/Th3 cytokines, and suppress inflammation by the mechanism of bystander suppression.

Hence, a significant degree of functional degeneracy exists in the recognition of self antigens by $\mathrm{T}$ cells.

\section{Bystander suppression}

The high frequency of activated, myelin-reactive $\mathrm{T}$ cells in the circulation and cerebrospinal fluid of patients with MS, an inflammatory disease of the white matter in the CNS, is consistent with the hypothesis that the disease is initiated by a microbial infection, as shown in Figure 1. In a genetically susceptible host, according to this model, infection by common pathogens stimulates the innate immune system, which activates the expression of costimulatory molecules by autoreactive $\mathrm{T}$ cells. At the same time, the presence of microbial antigens that cross-react with self antigens triggers these $T$ cells and leads to the autoimmune destruction of myelin and neuronal axons.

The observation of epitope spreading in the experimental autoimmune encephalomyelitis (EAE) model $(6,7)$ and the finding that self antigens elicit diverse $\mathrm{T}$ cell receptor repertoires have made approaches that target a single, antigen-reactive $\mathrm{T}$ cell theoretically untenable. In their place has emerged the concept of bystander suppression: Autoreactive Th2 or Th3 T cells, according to this model, are not lost quantitatively to negative selection but persist and migrate to the inflamed target organ, where they can be reactivated in an antigen-specific manner. However, cytokines produced by these cells then downregulate inflammation in the local milieu in an antigen-nonspecific mechanism (Figure 2) (8), thus suppressing autoimmune disease under most conditions. In this scenario, it should also be noted that high expression of costimulatory molecules in the sites of inflammation facilitates the epitope spreading (7), and allows a greater degree of $\mathrm{T}$ cell receptor degeneracy by driving clonal of potentially autoreactive $\mathrm{T}$ cells by lower affinity TCR/MHC peptide interactions (9).

\section{Altered peptide ligands}

It was recognized over a decade ago that the strength of signal delivered through the $\mathrm{T}$ cell receptor can determine which cytokines are secreted by the $\mathrm{T}$ cell (10). The cell apparently measures affinity in part by timing the engagement between the $\mathrm{T}$ cell receptor and the peptide/MHC complex. With longer engagement, a total, rather than partial, $\mathrm{T}$ cell receptor complex has time to form, and the extent of $\zeta$ chain phosphorylation increases correspondingly. Altered peptide ligand (APLs), which bind with low affinity to the $T$ cell receptor, weaken this signal. The ability of APLs to change the cytokine program of a $\mathrm{T}$ cell from a Th1 to a Th2 response was exploited first by Kuchroo and coworkers as a therapy for autoimmune disease (11). Using the murine EAE model of MS, these authors showed that APLs can activate IL-4 secretion by both encephalitogenic $\mathrm{T}$ cells and naive $T$ cell clones that crossreact with self antigens.

Injection of APLs is of clear therapeutic value in treating different models of EAE (12), and autoreactive human $\mathrm{T}$ cell clones can also be induced to secrete the antiinflammatory cytokines IL- 4 and TGF- $\beta$ after T cell receptor engagement by APLs (13, 14). However, as Ausubel et al. (15) noted, while APLs can induce Th2 cytokine secretion of MBP-reactive $T$ cells isolated from the peripheral blood $\mathrm{T}$ cells of MS patients, they can also induce a heteroclictic response in some patients, activating these MBP-reactive $T$ cells against the patient's own tissues. These data provide a strong rationale for the therapeutic use of APLs in patients with autoimmune disease. However, they also raise the issue that in some instances, highly degenerate $\mathrm{T}$ cell receptors can recognize APLs as self antigens.

A recently published phase II clinical trial testing an altered MBP p85-99 peptide confirms both of these conclusions. At the higher peptide dosage tested, two of seven MS patients developed remarkably high frequencies of MBP-reactive $T$ cells, and these 
responses were associated with significant increases in MRI-detectable lesions (16). In contrast, patients treated with lower doses of the APL showed no such disease flare-ups and may have indeed exhibited some degree of immune deviation toward increases in IL-4 secretion of MBP-reactive T cells $(17,18)$. Thus, APLs represent a classic double-edged sword. In our outbred population, given the high degree of degeneracy in the immune system, it is unclear whether it is possible to find APLs of self peptides that pose no risk of cross-reactivity with self.

\section{Glatiramer acetate}

An alternative approach to the use of a single APL is the administration of peptide mixtures that contain many different antigen specificities. Random copolymers that contain amino acids commonly used as MHC anchors and $\mathrm{T}$ cell receptor contact residues have been proposed as possible "universal APLs." GA is a random sequence polypeptide consisting of four amino acids - alanine (A), lysine $(\mathrm{K})$, glutamate $(\mathrm{E})$, and tyrosine $(\mathrm{Y})$, at a molar $\mathrm{A} / \mathrm{K} / \mathrm{E} / \mathrm{Y}$ ratio of $4.5: 3.6: 1.5: 1$ - and with an average length of 40-100 amino acids (19). Directly labeled GA binds efficiently to different murine H-2 I-A molecules, as well as to their human counterparts, the MHC class II DR molecules, but it does not recognize MHC class II DQ or MHC class I molecules in vitro (20).
In phase III clinical trials, GA, subcutaneously administered to patients with relapsing-remitting MS, decreases the rate of exacerbations and prevents the appearance of new lesions detectable by MRI (21). This represents perhaps the first successful use of an agent that ameliorates autoimmune disease by altering signals through the TCR.

A “universal antigen” containing multiple epitopes would be expected to induce proliferation in vitro in naive $T$ cells from the circulation, due to its expected high degree of crossreactivity with other peptide antigens. Indeed, GA induces strong MHC class II DR-restricted proliferative responses in T cells isolated from MS patients or from healthy controls (22). In most patients, daily injection with GA causes a striking loss of responsiveness to this polymer antigen, accompanied by greater secretion of IL-5 and IL- 13 by $\mathrm{CD}^{+} \mathrm{T}$ cells, indicating a shift toward a Th2 response (22-26). The paper by Karandikar and coworkers (2) in this issue of the JCI confirms that this treatment causes a shift by $\mathrm{CD}^{+} \mathrm{T}$ cells toward a Th 2 or Th3 phenotype, as judged by increased levels of TGF- $\beta$ mRNA and cell-associated IL-4. In addition, the surviving GA-reactive T cells exhibit a high degree of degeneracy, as measured by their ability to cross-react with a large variety of peptides represented in a combinatorial library (23).
Thus, in vivo administration of GA induces highly cross-reactive $\mathrm{CD} 4^{+} \mathrm{T}$ cells that are immune-deviated to secrete Th2 cytokines (Figure 2). We have proposed that GA-induced migration of highly cross-reactive Th2 (and perhaps Th3) cells to sites of inflammation allows their highly degenerate $T$ cell receptors to contact self antigens, which they recognize as weak agonists, much like APLs. These $T$ cells then apparently secrete suppressive, Th2/Th3 cytokines, thus restricting local inflammation (22). We concluded that, through this form of bystander suppression, GA can effect an immune deviation to a Th2 response and may prove useful in a variety of autoimmune disorders.

\section{$\mathrm{CD8}^{+} \mathrm{T}$ cells}

In their present report (2), Karandikar and coworkers have found that MHC class I-restricted $\mathrm{CD}^{+} \mathrm{T}$ cells in untreated MS patients respond weakly to GA. Treatment with GA stimulates these responses, restoring them to levels observed in healthy individuals. Because GA therapy significantly increases IFN- $\gamma$ expression by CD $8 \mathrm{~T}$ cells, the authors propose that these cells regain an ability to suppress myelin-reactive Th 1 cells (Figure 3 ).

The significance of decreased $\mathrm{CD}^{+}$ $T$ cell responsiveness to GA in patients with MS is not clear, however. GA can be viewed as a nonspecific TCR agonist, perhaps similar to an anti-CD3

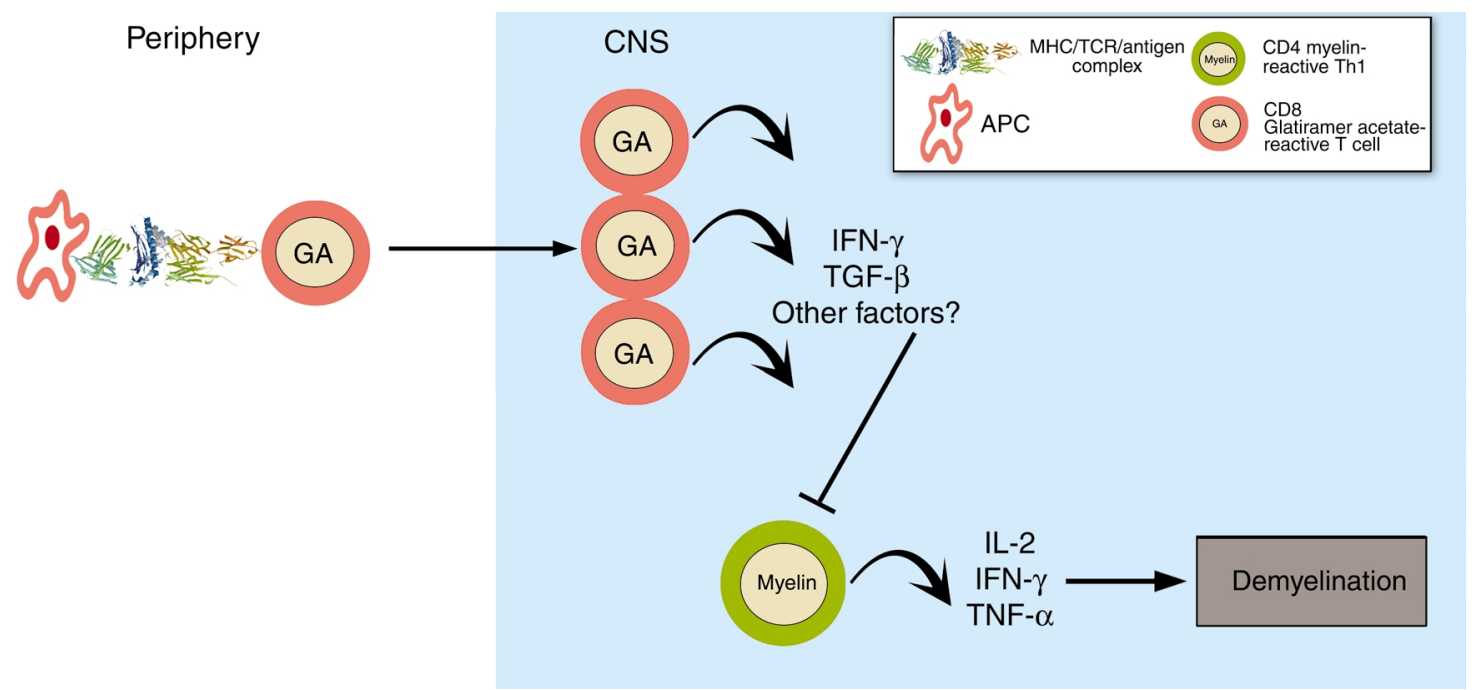

\section{Figure 3}

The CD8+ T cell model of action by GA. MHC class I restricted CD8 $8^{+} \mathrm{T}$ cell responses to GA, which are significantly lower at baseline in MS patients than in healthy controls, increase significantly following treatment, as seen in the greater number of IFN- $\gamma$-positive, GA responsive CD $8^{+} \mathrm{T}$ cells. GA treatment is proposed to restore $\mathrm{CD} 8^{+}$suppressor function, which is dependent upon IFN- $\gamma$ secretion. The mechanism for $\mathrm{CD} 8^{+}$suppression of the immune response is unknown. Shown in the figure is a $\mathrm{CD} 8^{+} \mathrm{T}$ cell migrating to the CNS, regulating a $\mathrm{CD} 4^{+}$autoreactive $\mathrm{T}$ cell. 
$\mathrm{mAb}$, which would be expected to bind equally to all TCR complexes. Thus, the decreased response by $\mathrm{CD}^{+} \mathrm{T}$ cells to GA may represent a general nonresponsiveness of MHC class I-restricted CD8 T cells in patients with MS. This finding harks back to earlier analyses of peripheral blood in patients with MS, which also revealed a modest decrease in $\mathrm{CD}^{+} \mathrm{T}$ cell numbers. Alternatively, GA may target pathological subpopulations of CD8 T cells, preventing destruction of the target tissue. Indeed, we have recently found evidence for the existence of what appear to be pathologic CD8 cells in the circulation of patients with MS. We examined the response of highly purified $\mathrm{CD}^{+} \mathrm{T}$ cells from MS patients to TCR cross-linking in the presence or absence of a costimulatory signal provided by anti-CD28. CD8 ${ }^{+}$ $\mathrm{T}$ cells from MS patients, but not from normal subjects, secrete more lymphotoxin and TNF- $\alpha$ in response to T cell receptor cross-linking (G. Buckle and D. Hafler, unpublished observations). The ability of these patients' $\mathrm{CD}^{+} \mathrm{T}$ cells to secrete high amounts of lymphotoxin, independent of costimulation, suggests that such cells could be directly involved in CNS damage in MS and might not act through a separate population of $\mathrm{CD} 4^{+} \mathrm{Th} 1$ cells, as suggested by Karandikar et al. (2).

\section{Conclusions}

How can these changes in $\mathrm{CD}^{+}$ responses be reconciled with what has been demonstrated regarding GA's effect on $\mathrm{CD}^{+} \mathrm{T}$ cells? To address this question, it will be essential to determine how the $\mathrm{CD}^{+} \mathrm{GA}$-reactive $\mathrm{T}$ cells identified by Karandikar et al. (2) contribute to the disease process in EAE and MS. As the authors suggest, these cells may represent regulatory $\mathrm{T}$ cells whose activity and ability to secrete IFN- $\gamma$ can be enhanced by GA treatment. Alternatively, they may be activated, lymphotoxin-secreting cytotoxic $\mathrm{T}$ cells whose capacity to mediate demyelination is somehow inhibited by GA.
Perhaps different populations of $\mathrm{CD}^{+}$cells in these individuals exhibit different functions at the same time. Indeed, the division of $\mathrm{CD} 8^{+} \mathrm{T}$ cells into functionally discrete $\mathrm{CD} 28^{+}$ and CD28- populations has been known for some time (27). It appears that GA may have more than one mechanism of action, inducing different effects in $\mathrm{CD}^{+}$and $\mathrm{CD}^{+} \mathrm{T}$ cells that work in separate but parallel immune pathways. Clearly, further studies are required on the effects of GA on the various $\mathrm{CD}^{+} \mathrm{T}$ cell populations in patients with MS.

\section{Acknowledgments}

I would like to thank our laboratory for helpful comments reading this Commentary. Disclosure: The author has received grant support from Teva Neuroscience.

1. Wucherpfennig, K.W., and Strominger, J.L. 1995 Molecular mimicry in T cell-mediated autoimmunity: viral peptides activate human $\mathrm{T}$ cell clones specific for myelin basic protein. Cell. 80:695-705

2. Karandikar, N.J., et al. Glatiramer acetate (Copaxone) therapy induces $\mathrm{CD}^{+} \mathrm{T}$ cell responses in patients with multiple sclerosis. J. Clin. Invest. 109:641-649. DOI:10.1172/JCI200215198.

3. Ota, K., et al. 1990. T-cell recognition of an immunodominant myelin basic protein epitope in multiple sclerosis. Nature. 346:183-187.

4. Ausubel, L.J., et al. 1996. Complementary mutations in an antigenic peptide allow for crossreactivity of autoreactive T-cell clones. Proc. Natl. Acad. Sci. USA. 93:15317-15322.

5. Hemmer, B., et al. 1997. Identification of high potency microbial and self ligands for a human autoreactive class II-restricted T cell clone. J. Exp. Med. 185:1651-1659.

6. Lehmann, P.V., et al. 1992. Spreading of T-cell autoimmunity to cryptic determinants of an autoantigen. Nature. 358:155-157.

7. Miller, S.D., et al. 1995. Blockade of CD28/B7-1 interaction prevents epitope spreading and clinical relapses of murine EAE. Immunity. 3:739-745.

8. Chen, Y., et al. 1994. Regulatory T cell clones induced by oral tolerance: suppression of autoimmune encephalomyelitis. Science. 265:1237-1240.

9. Anderson, D.E., et al. 1997. Weak peptide agonists reveal functional differences in B7-1 and B72 costimulation of human $\mathrm{T}$ cell clones. $J$. Immunol. 159:1669-1675.

10. Evavold, B.D., and Allen, P.M. 1991. Separation of IL-4 production from Th cell proliferation by an altered $\mathrm{T}$ cell receptor ligand. Science. 252:1308-1310.

11. Nicholson, L.B., et al. 1997. A T cell receptor antagonist peptide induces $\mathrm{T}$ cells that mediate bystander suppression and prevent autoim- mune encephalomyelitis induced with multiple myelin antigens. Proc. Natl. Acad. Sci. USA. 94:9279-9284

12. Brocke, S., et al. 1996. Treatment of experimental encephalomyelitis with a peptide analogue of myelin basic protein. Nature. 379:343-346.

13. Windhagen, A., et al. 1995. Modulation of cytokine patterns of human autoreactive $\mathrm{T}$ cell clones by a single amino acid substitution of their peptide ligand. Immunity. 2:373-380.

14. Ausubel, L.J., Krieger, J.I., and Hafler, D.A. 1997. Changes in cytokine secretion induced by altered peptide ligands of myelin basic protein peptide 85-99. J. Immunol. 159:2502-2512.

15. Ausubel, L.J., Bieganowska, K.D., and Hafler, D.A. 1999. Cross-reactivity of T-cell clones specific for altered peptide ligands of myelin basic protein. Cell. Immunol. 193:99-107.

16. Bielekova, B., et al. 2000. Encephalitogenic potential of the myelin basic protein peptide (amino acids 83-99) in multiple sclerosis: results of a phase II clinical trial with an altered peptide ligand. Nat. Med. 6:1167-1175.

17. Kappos, L., et al. 2000. Induction of a nonencephalitogenic type $2 \mathrm{~T}$ helper-cell autoimmune response in multiple sclerosis after administration of an altered peptide ligand in a placebo-controlled, randomized phase II trial. The Altered Peptide Ligand in Relapsing MS Study Group. Nat. Med. 6:1176-1182.

18. Crowe, P.D., et al. 2000. NBI-5788, an altered MBP83-99 peptide, induces a T-helper 2-like immune response in multiple sclerosis patients. Ann. Neurol. 48:758-765.

19. Teitelbaum, D., et al. 1971. Suppression of experimental allergic encephalomyelitis by a synthetic polypeptide. Eur. J. Immunol. 1:242-248.

20. Fridkis-Hareli, M. and Strominger, J.L. 1998 Promiscuous binding of synthetic copolymer 1 to purified HLA-DR molecules. J. Immunol. 160:4386-4397.

21. Johnson, K.P., et al. 1995. Copolymer 1 reduces relapse rate and improves disability in relapsingremitting multiple sclerosis: results of a phase III multicenter, double-blind, placebo-controlled trial. Neurology. 45:1268-1276.

22. Duda, P.W., et al. 2000. Human and murine CD4 $T$ cell reactivity to a complex antigen: recognition of the synthetic random polypeptide glatiramer acetate. J. Immunol. 165:7300-7307.

23. Duda, P.W., et al. 2000. Glatiramer acetate (Copaxone) induces degenerate, Th2-polarized immune responses in patients with multiple sclerosis. J. Clin. Invest. 105:967-976.

24. Neuhaus, O., et al. 2000. Multiple sclerosis: comparison of copolymer-1-reactive $\mathrm{T}$ cell lines from treated and untreated subjects reveals cytokine shift from T helper 1 to T helper 2 cells. Proc. Natl. Acad. Sci. USA. 97:7452-7457.

25. Gran, B., et al. 2000. Mechanisms of immunomodulation by glatiramer acetate. $\mathrm{Neu}$ rology. 55:1704-1714.

26. Farina, C., et al. 2001. Treatment of multiple sclerosis with Copaxone (COP): Elispot assay detects COP-induced interleukin-4 and interferongamma response in blood cells. Brain. 124:705-719.

27. Turka, L.A., et al. 1990. CD28 is an inducible T cell surface antigen that transduces a proliferative signal in CD3+ mature thymocytes. J. Immunol. 144:1646-1653. 